\title{
DIVERSIDAD Y PATRONES REPRODUCTIVOS DE QUIRÓPTEROS EN UNA ÁREA URBANA DE LIMA, PERÚ.
}

José Luis Mena ${ }^{1}$ y Martha Williams de Castro $^{2}$

\section{Resumen}

Resultan particularmente escasos los estudios sobre vertebrados en áreas que soportan la acción modificadora del hombre. Los estudios sobre la biología reproductiva de murciélagos neotropicales indican que la mayoría se reproducen estacionalmente, de allí que surgió la necesidad de documentar este comportamiento en el campus de la Universidad Nacional Agraria La Molina, área urbana de la ciudad de Lima, Perú. Las colectas se efectuaron de octubre de 1995 a septiembre de 1996 con el objeto de reconocer los patrones reproductivos de las especies. Se capturó un total de 180 individuos, registrándose cuatro especies pertenecientes a las familias Phyllostomidae y Vespertilionidae. Las especies más abundantes fueron Glossophaga soricina y Artibeus fraterculus. Para estas especies, se confirman los patrones postulados de poliestría bimodal. Los resultados revelan que ambas especies siguen este patrón y que las hembras de $G$. soricina y A. fraterculus por lo menos tienen un período de estro post parto por año.

Palabras clave: Quirópteros, patrones reproductivos, áreas urbanas.

\begin{abstract}
There are particularly few vertebrate studies in areas that support man's modifying action. Studies on the reproductive biology of Neotropical bats indicate that the majority reproduce seasonally. This lead us to document this behaviour at the La Molina National Agrarian University campus, located in an urban area of the city of Lima, Peru. In order to recognise the reproductive patterns of the species, bats were captured from October 1995 to September of 1996. A total of 180 individuals was captured, comprising 4 species, pertaining to the Phyllostomidae and Vespertilionidae families. The most abundant species were Glossophaga soricina and Artibeus fraterculus. For these species, bimodal polyestric patterns are reported. Results reveal that both species follow this pattern and that the females of G. soricina . and $A$. fraterculus have at least one post - partum estrous period per year.
\end{abstract}

Key words: Chiroptera, reproductive patterns, urban areas.

\section{Introducción}

Generalmente, los estudios biológicos se efectúan en áreas silvestres prístinas o poco alteradas por el hombre con el fin de aislar el efecto humano en el desarrollo de las investigaciones. Por ejemplo, la mayoría de los estudios en quirópteros han sido efectuados en el bosque lluvioso (Ascorra et al., 1989; Ascorra et al., 1993; Ascorra et al., 1996; Solari et al., 1998; Voss \& Emmons, 1996; Wilson et al., 1996). Contrariamente, son muy escasos los estudios en áreas que soportan la acción modificadora del hombre, sobretodo, en zonas urbanas. Destacan, sin embargo, algunos estudios realizados en la ciudad de Lima (Perú) y Rosario (Argentina), en grupos como aves (Pacheco et al., 1990) y quirópteros (Ortiz de la Puente, 1951; Romano et al., 1999). En lo que respecta a este último grupo, es necesario destacar el estudio realizado ya hace medio siglo por Ortiz de la Puente (1951), reportando un total de 14 especies para Lima y alrededores.
Los quirópteros tropicales tienen un gran potencial como indicadores de niveles de perturbación de hábitat y ofrecen una amplia visión de la salud de un ecosistema debido a que explotan diferentes recursos tróficos (Fenton et al., 1992). Juntamente con las aves, destacan como importantes agentes en la dispersión de semillas en bosques tropicales (Howe \& Smallwood, 1982). Así mismo, debido a la dieta insectívora de algunas especies, pueden ser considerados controladores naturales ya que consumen enormes cantidades de insectos (Eisenberg \& Redford, 1999; Emmons \& Feer, 1997; Romano et al., 1999), muchos de los cuales podrían ser plagas de cultivos agrícolas.

El estudio integral de los murciélagos en ambientes urbanos puede ayudar a eliminar algunos prejuicios contra este grupo de mamíferos. Por ejemplo, en la ciudad de Rosario (Argentina), se ha registrado el valioso aporte de $T$. brasiliensis al control de insectos: un total de 209 a $385 \mathrm{Kg}$. de insectos nocturnos consumidos en un período de 5

\footnotetext{
${ }^{1}$ Laboratorio de Fisiología Animal y Biorremediación. Departamento de Biología, Universidad Nacional Agraria La Molina . Apartado 456. Lima 100. Correo electrónico: jlmena@terra.com.pe

${ }^{2}$ Profesora Principal, Laboratorio de Fisiología Animal y Biorremediación. Departamento de Biología, Universidad Nacional Agraria La Molina . Apartado 456. Lima 100. Correo electrónico: Mwilliams@lamolina.edu.pe
} 
meses revelan el importante rol que desempeña esta especie en dicha ciudad (Romano et al., 1999). Por tales razones, en algunos países existen legislaciones específicas para su protección (Racey, 1982; Romano et al., 1999)

Los patrones de reproducción, no sólo de murciélagos sino también de mamíferos (en sistemas frugívoro - planta), son claramente estacionales, sobretodo en el bosque tropical; de modo que los picos en la abundancia de frutos generalmente coinciden o son influenciados por el incremento en las lluvias (Frankie et al, 1974; Hilty, 1980). Estudios sobre la biología reproductiva de murciélagos neotropicales indican que la mayoría se reproducen estacionalmente (Fleming et al, 1972; Wilson, 1979), siendo poliestras la mayoría de especies (Bonaccorso, 1979; Bradbury \& Vehrencamp, 1976; Fleming et al, 1972; Wilson, 1979). Este patrón estacional de reproducción corresponde a incrementos estacionales en la abundancia alimenticia, tanto para frugívoros como para insectívoros (August \& Baker, 1982). Esta hipótesis está basada principalmente en estudios desarrollados en Costa Rica y Panamá (Fleming et al., 1972; Humphrey \& Bonaccorso, 1979; LaVal \& Fitch, 1977). En el Perú, se han registrado algunos patrones estacionales (Graham, 1989), pero sobretodo en bosque lluvioso.

En el caso particular de la ciudad de Lima y posiblemente para la mayoría de la costa peruana, la precipitación es casi insignificante. Por ejemplo, se conoce que la precipitación anual promedio de 18 años ha sido de sólo $46 \mathrm{~mm}$., sin embargo, se pueden definir dos estaciones más o menos claras: una estación cálida y una estación fría en donde las nubes forman una cobertura densa (Holdridge, 2000). Debido a que en Lima no existe una estacionalidad basada en precipitación, la cual es conocida como determinante para la producción de frutos y por ende del comportamiento reproductivo de quirópteros; es interesante evaluar los factores que últimamente determinan la reproducción de estas especies en este tipo de ambiente, especialmente porque se trata de una zona urbana.

El objetivo principal de este estudio fue conocer la diversidad de quirópteros que habitan ambientes urbanos, específicamente en el campus de la Universidad Nacional Agraria La Molina (UNALM). Así mismo, reconocer patrones reproductivos de las especies tomando en cuenta la fenología de las plantas y las condiciones climáticas durante el período de evaluación.

\section{Materiales y métodos}

El presente estudio se llevó a cabo en el campus de La Universidad Nacional Agraria La Molina, ubicada en el departamento de Lima, Perú $\left(12^{\circ} 05^{\prime}\right.$ S, $76^{\circ} 57^{\prime}$ O) La zona presenta una temperatura anual promedio de $20^{\circ} \mathrm{C}$, una humedad relativa anual promedio de
$81 \%$ y una precipitación total anual de $10 \mathrm{~mm}$. El área de muestreo comprendió al jardín botánico de la universidad y los sótanos de algunos edificios que servían como refugios para los murciélagos. La vegetación presente en la UNALM (jardín botánico, parques circundantes, y campos de cultivo) constituye una fuente de alimento que es aprovechada por los murciélagos, no sólo frugívoros sino también insectívoros que se alimentan de insectos que frecuentan dichas áreas. Entre las principales especies vegetales susceptibles de ser visitadas por murciélagos se encuentran: Morus nigra, Ficus carica, Ficus obtusifolia, Fenix canadiense, Solanum mauritanum.

La evaluaciones se realizaron una vez por mes durante el período comprendido entre octubre de 1995 y septiembre de 1996. Se usaron redes de neblina (usualmente de $6 \mathrm{~m}$ de largo por $2 \mathrm{~m}$ de alto), colocadas de 1-3 metros sobre el nivel del suelo. Las redes fueron abiertas a las 18:00 p.m. y revisadas a intervalos de 1 a 2 horas, hasta las 12:00 a.m. El esfuerzo total comprendió 30 redes noche acumuladas durante los 12 meses de trabajo. Los animales capturados fueron identificados, pesados y guardados en bolsas de tela durante 1 hora con la finalidad de colectar muestras de heces y posteriormente fueron liberados.

A los individuos capturados se les tomó los siguientes datos: especie, hora y localización de captura, sexo, peso, longitud de antebrazo, edad (juveniles y adultos), estado reproductivo de hembras por observación de preñez, y de machos al observar la posición de los testículos (escrotales o abdominales). La edad se determinó a través de características de pelo, masa corporal y fusión de epifisis metacarpales. Para la determinación de especies se utilizó la clave de identificación de Ortiz de la Puente (1951), y Emmons \& Feer (1997).

\section{Resultados}

Riqueza de Especies

Se capturaron un total de 180 individuos, registrándose cuatro especies pertenecientes a las familias Phyllostomidae y Vespertilionidae (Tabla 1). Las especies más abundantes dentro de la UNALM fueron Glossophaga soricina y Artibeus fraterculus. Se reporta también a Tadarida brasiliensis (Molossidae) como una especie de ambientes urbanos, pero registrada fuera del área de estudio, en el campus de la Universidad Nacional Mayor de San Marcos (Luna, com. pers.).

Glossophaga soricina es una especie ampliamente distribuida, desde el norte de México hasta el norte de Argentina y sudeste de Brasil; y en nuestro país se le encuentra en casi todas las regiones (Eisenberg \& Redford, 1999; Nowak, 1999). Su dieta consiste principalmente de fruta, insectos y probablemente néctar, pero presenta una alta variación espacial. 
Tabla 1. Murciélagos capturados en ambientes urbanos en la zona de Lima. Los números corresponden a individuos capturados en la Universidad Nacional Agraria La Molina, Perú.

\begin{tabular}{|c|c|c|}
\hline Especie & $\begin{array}{c}\mathrm{N}^{\circ} \\
\text { capturas }\end{array}$ & Gremio \\
\hline \multicolumn{3}{|l|}{ Familia Phyllostomidae } \\
\hline \multicolumn{3}{|l|}{ Subfamilia Glossophaginae } \\
\hline Anoura geoffroyi Gray, 1853 & 1 & $\begin{array}{l}\text { Nectarívoro - } \\
\text { Insectívoro }\end{array}$ \\
\hline $\begin{array}{l}\text { Glossophaga soricina } \\
\text { Geoffroyi, } 1818\end{array}$ & 105 & $\begin{array}{l}\text { Nectarívoro - } \\
\text { Insectívoro }\end{array}$ \\
\hline \multicolumn{3}{|l|}{ Subfamilia Stenodermatinae } \\
\hline $\begin{array}{l}\text { Artibeus fraterculus Anthony, } \\
1924\end{array}$ & 73 & Frugívoro \\
\hline \multicolumn{3}{|l|}{ Familia Vespertilionidae } \\
\hline $\begin{array}{l}\text { Lasiurus blossevillii Lesson \& } \\
\text { Carmot, } 1826\end{array}$ & 1 & Insectívoro \\
\hline \multicolumn{3}{|l|}{ Familia Molossidae } \\
\hline $\begin{array}{l}\text { Tadarida brasiliensis (I. } \\
\text { Geoffroy, 1824) }\end{array}$ & $\mathrm{r}^{*}$ & Insectívoro \\
\hline
\end{tabular}

r* : Ejemplar colectado en el campus de la Universidad Nacional Mayor de San Marcos, Lima Perú (Luna com. pers.)

Ha sido reportada como polinizador de Luchea sp. en Brasil (Nowak, 1999). La evaluación de algunos tractos digestivos de individuos de G. soricina revela que se alimenta de partes de flores (polen de maíz) e insectos. Fue reportada como una especie frecuente para la ciudad de Lima (Ortiz de la Puente, 1951)

Artibeus fraterculus otra de las especies abundantes en la UNALM, ocurre en los bosques occidentales de Ecuador y Perú (Eisenberg \& Redford, 1999). En nuestro país se conocen registros de Tumbes, Cajamarca, Ancash y Lima (Patterson et al., 1992) y fue reportada como frecuente para Lima por Ortiz de la Puente (1951). El género Artibeus es principalmente frugívoro (Gardner, 1977; Nowak, 1999); algunos individuos fueron capturados con frutos de Ficus sp. y también se encontraron gran cantidad de semillas de esta planta en los refugios visitados.

Anoura geoffroyi según los eventos de muestreo parece ser una especie poco abundante, ocurre desde México hasta Bolivia y el sureste de Brasil, Trinidad (Eisenberg \& Redford, 1999; Nowak, 1999). Su ocurrencia ha sido registrada para nuestro país en el lado amazónico y en el Pacífico (Koopman, 1978). Esta especie fue registrada en Lima en los años 1942 y 1950 (Ortiz de la Puente, 1951); el presente articulo constituye un registro actualizado de su presencia en zonas urbanas. Posee hábitos insectívoros en ciertas estaciones del año (Eisenberg \& Redford, 1999; Emmons \& Feer, 1997); Ortiz de La Puente (1951) registra contenidos estomacales con restos de coleópteros de la familia Nitidulidae.

Lasiurus blossevilli al igual que la especie anterior fue registrada sólo en una oportunidad; presenta un rango de distribución muy amplio desde la Columbia Británica a través de Utah y México a Chile y Argentina (Eisenberg \& Redford, 1999; Nowak, 1999). Se conocen registros para la ciudad de Lima y Callao. Es considerada una especie rara para la Lima (Ortiz de la Puente, 1951). El género Lasiurus ha sido estudiado ampliamente en Norteamérica exhibiendo migración estacional al igual que Tadarida brasiliensis (Eisenberg \& Redford, 1999); ambos géneros son principalmente insectívoros (Eisenberg \& Redford, 1999; Emmons \& Feer, 1997; Nowak, 1999) y en Sudamérica se sabe poco de sus hábitos.

En la Figura 1 se muestra los esfuerzos de captura durante el período de estudio. La conducta estable de la curva conduce a pensar que se ha evaluado posiblemente a todas las especies de murciélagos de la UNALM. En comparación a la lista para Lima presentada por Ortiz de la Puente (1951) es un número menor pero dadas las características de refugio y alimentación que presenta la UNALM puede ser posible encontrar otras especies.

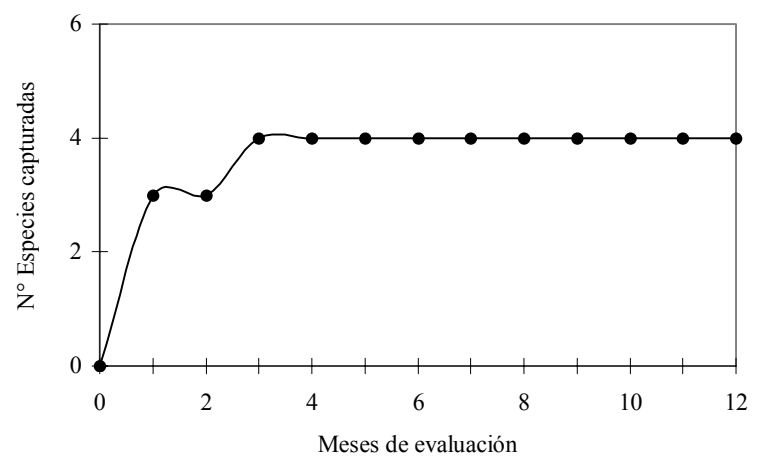

Figura 1. Curva de acumulación de especies de murciélagos capturados durante el período de evaluación octubre 95-septiembre 96 en la Universidad Nacional Agraria La Molina, Perú

Los gremios tróficos muestran el diferente uso de los recursos que hacen los murciélagos (Tabla 1), así como la presencia de distintos microhábitat's que permiten prosperar a las especies registradas en éste estudio. A. fraterculus, $G$. soricina y $A$. geoffroyi fueron capturados en sótanos $\mathrm{y}$ techos de construcciones usadas como laboratorios y oficinas. $L$. blossevilli fue capturada en áreas abiertas del jardín botánico de la UNALM, esta especie frecuentemente usa ramas de árboles y construcciones humanas como lugares de descanso (Nowak,1999).

\section{Patrones reproductivos}

El análisis de patrones reproductivos correspondió a las especies con mayores registros: A. fraterculus y G. Soricina. Se confirman los patrones postulados de 
poliestría bimodal (Fleming et al., 1972; Nowak, 1999; Wilson, 1979). Los resultados revelan que ambas especies siguen este patrón y que las hembras de $G$. soricina y $A$. fraterculus por lo menos tienen un período de estro post parto por año.

Los períodos de preñez y lactación claramente indican que dos picos de nacimientos ocurren anualmente en $A$. fraterculus, donde el primer período de nacimientos se reporta para febrero, mes más caluroso de la estación de verano; el segundo período se registra en mayo (Figura 2). En G. soricina el patrón es similar, pero los nacimientos ocurren en enero y en abril (Figura 3).

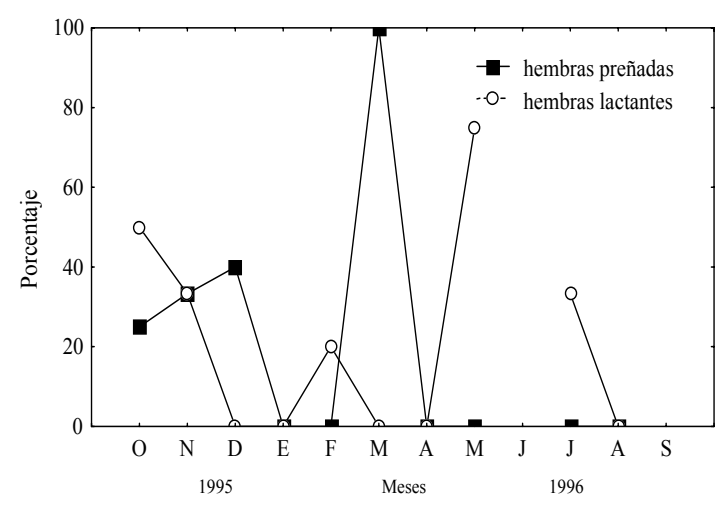

Figura 2. Condición reproductiva del murciélago frugívoro Artibeus fraterculus. Porcentajes de preñez son basados en condiciones macroscópicas.

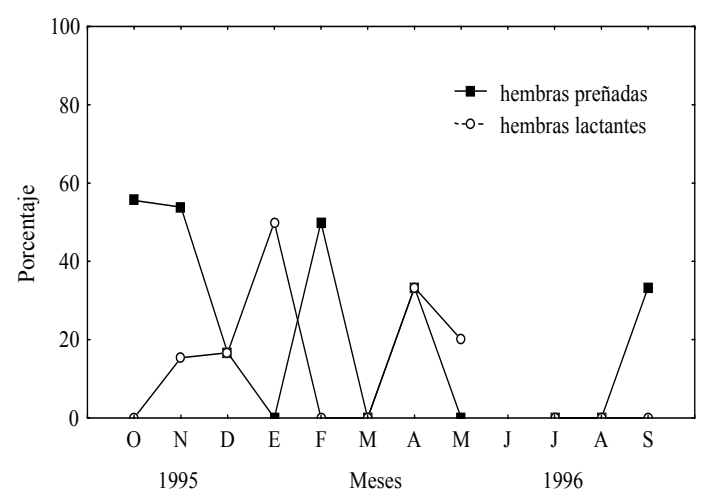

Figura 3. Condición reproductiva del murciélago nectarívoro insectívoro Glossophaga soricina. Porcentajes de preñez son basados en condiciones macroscópicas

Reportamos también un interesante patrón en la conducta reproductiva de A. fraterculus y $G$. soricina, el cual permite especular sobre la dieta de ambas especies. En la Figura 4 se puede observar un patrón, donde el pico de nacimientos para $G$. soricina es seguido por un pico de nacimientos para un frugívoro como A. fraterculus. Posiblemente este patrón corresponda al desarrollo fenológico de algunas plantas que pueden ser principales fuentes de alimento. Así mismo, podría sugerirse que $G$. soricina presente una alimentación basada en flores, principalmente por que los nacimientos en esta especie ocurren antes del pico de reproducción de los frugívoros, es decir cuando debe haber una proporción mayor de plantas con flores. Por su parte, $A$. fraterculus, una especie de hábitos eminentemente frugívoros, debe adaptar su mayor nivel de nacimientos al período en el cual existe una mayor abundancia de frutos.

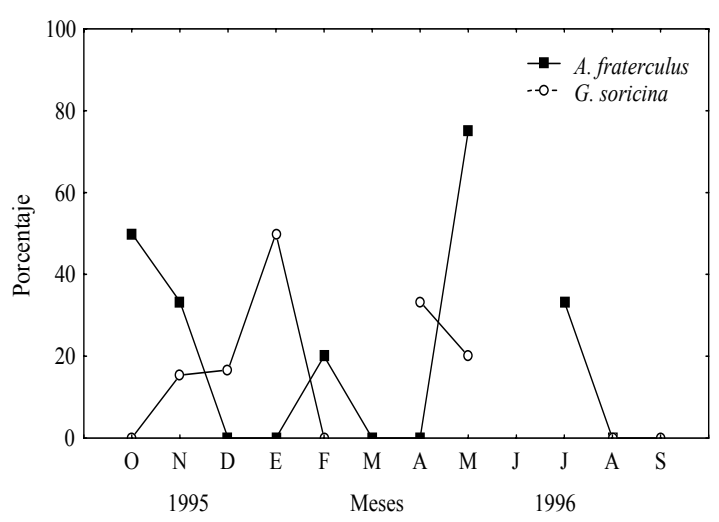

Figura 4. Relación entre los estados de lactancia de Artibeus fraterculus y Glossophaga soricina, mostrando una posible relación con la fenología de plantas en concordancia a los hábitos alimentarios de ambas especies.

\section{Discusión}

La presencia de cuatro especies de murciélagos con variados requerimientos alimenticios revelan que la UNALM ofrece una importante fuente de recursos y refugio. Casos de competencia por alimento para el caso de $A$. geoffroyi y $G$. soricina deberían ser comprobados. Es posible que otras especies estén presentes en la zona, sobretodo aquellas que ocurren en áreas urbanas, como por ejemplo T. brasiliensis que fue registrada en la ciudad de Rosario, Argentina (Romano et al., 1999) y también en el centro de Lima (Luna com. pers.). Desmodus rotundus podría encontrar una buena fuente de alimento en el ganado presente en la zona y su ocurrencia podría ser probable.

En el registro actual de ocurrencia de murciélagos para la ciudad de Lima se pueden mencionar las 5 especies capturadas en áreas urbanas (incluyendo $T$. brasiliensis). Después del último reporte sobre quirópteros limeños hecho por Ortiz de la Puente (1951) es posible que la diversidad haya disminuido. Los reportes hechos por Ortiz de la Puente corresponden a posiblemente 14 especies, en su mayoría capturadas en el actualmente poblado cerro el Agustino (Lima). Tres de ellas son consideradas 
frecuentes en Lima y alrededores: G. soricina, $A$. fraterculus $y$ Amorphochilus schnablii, las dos primeras fueron abundantes en la UNALM y la última posiblemente también se encuentre presente. Para la ciudad de Rosario (Argentina) se ha reportado un total de 7 especies de las familias de insectívoros Molossidae y Vespertilionidae (Romano et al., 1999); la presencia de la familia Phyllostomidae la cual presenta miembros con una diversidad de hábitos alimentarios y con un alto número de especies dentro de los quirópteros del neotrópico (Voss \& Emmons, 1996), posiblemente sea un indicativo de una mayor oferta de recursos en la UNALM.

La relación entre una mayor abundancia de alimentos (energía disponible) y los ciclos reproductivos en murciélagos han sido ampliamente comprobados (Fleming et al., 1972; Wilson, 1979; Dinerstein, 1986; Wilson et al., 1991; Autino \& Barquez, 1993); sin embargo, en zonas donde los niveles alimenticios permanecen distributivamente constantes a través del año, la reproducción puede ser acíclica (Fleming et al., 1972), pero este no ha sido el caso en la UNALM a pesar de que se trata prácticamente de una zona sin una precipitación considerable. Nuestros resultados demuestran una estacionalidad en la reproducción.

En la poliestría bimodal, la reproducción está restringida a una época del año, con dos picos de nacimientos que se producen en los meses energéticamente más favorables; lo cual es una característica de los filostómidos en general (Autino \& Barquez, 1993). Nuestros resultados concuerdan con estos patrones y con los descritos específicamente para los géneros Glossophaga y Artibeus, por lo que es evidente de que las especies encontradas deben presentar un estro postparto. Eventos como los reportados en $A$. jamaicensis con un segundo estro postparto y un desarrollo retrasado hasta la próxima estación húmeda (Wilson, 1979) podrían ser evaluados en $A$. fraterculus.

Wilson (1979) y Nowak (1999) sugieren que Glossophaga soricina es poliestra y con un patrón bimodal en la mayoría de localidades evaluadas, este patrón ha sido confirmado en nuestro estudio. Para $G$. soricina se ha reportado evidencias de reproducción para Sudamérica durante todo el año, pero con picos en diciembre - febrero y julio - agosto (Nowak, 1999). El ciclo de estro comprende 22-26 días y la gestación probablemente de 2-3 meses (normalmente es una sola cría). Nosotros reportamos el inicio del ciclo reproductivo para $G$. soricina durante el mes de enero (Figura $\mathrm{N}^{\circ}$ 2), y esto concuerda con lo reportado para otras áreas de Sudamérica (Fleming et al., 1972; Wilson, 1979; Nowak, 1999). Sin embargo, el segundo pico de nacimientos (mes de abril) es más pronto que lo encontrado en otras localidades donde frecuentemente ocurre en julio - agosto. Es necesario destacar que entre enero $y$ abril se registran las temperaturas más cálidas del año (sobretodo en enero y febrero); posiblemente, esta especie aproveche los meses más calurosos del año para reproducirse. Los porcentajes de preñez o lactancia encontrados en este estudio concuerdan con los reportados por Graham (1989)

Con respecto $A$. fraterculus no existen datos completos sobre su reproducción (por lo menos a nivel local), sin embargo Graham (1989) registra algunos datos de preñez de $A$. fraterculus para el Perú, los cuales son concordantes con lo encontrado en nuestro estudio; es decir con un inicio del ciclo que corresponde al mes de febrero y con un segundo pico de nacimientos que posiblemente ocurre durante el mes de mayo (Figura 3). En A. jamaicensis el primer pico de nacimientos ocurre también en febrero y el segundo comienza en julio y agosto, con el mismo patrón de estro postparto y lactación, con un período de gestación de 3.5 a 4 meses (Wilson et al., 1991). Según los patrones observados para $A$. fraterculus (Figura 3) este período podría ser unos tres meses.

Dentro de la conducta reproductiva de loa quirópteros, también es interesante tomar en cuenta algunas variaciones en el ciclo observadas principalmente entre algunas especies de Stenodermatinos, donde se ha registrado que pueden variar su conducta reproductiva poliestra bimodal a monoestra. Este fenómeno ha sido reportado en poblaciones ubicadas en los límites de distribución geográfica, tal como ha sido el caso en Sturnira erythromos y Sturnira lilium, específicamente en la parte más austral de su distribución (Autino \& Barquez, 1993). En tal sentido, hay que considerar que A. fraterculus posiblemente tenga su límite de distribución sur en el ámbito de la zona de estudio, en este caso en la costa central del Perú (Eisenberg \& Redford, 1999). Un estudio filogenético realizado por Patterson et al. (1992) en esta especie, demuestra que existen poblaciones perífericas y aisladas en San Bartolomé, ubicado en la sierra de Lima a 1600 msnm. Posiblemente la UNALM podría constituir un sugestivo punto de partida para comprobar si el mencionado cambio en la conducta reproductiva podría observarse también en $A$. fraterculus, ya que a pesar de que nuestros resultados revelan un patrón bimodal, es necesario que sea confirmado también en otras poblaciones cercanas al área de estudio.

A. fraterculus es un poco menor en tamaño que su pariente $A$. jamaicensis con pesos de $36-43$ y $40-42$ gramos respectivamente (Eisenberg \& Redford, 1999). Posiblemente, algunas características fisiológicas encontradas en $A$. jamaicensis puedan presentarse también en $A$. fraterculus, aunque hay que considerar que filogenéticamente son dos especies no muy emparentadas (Patterson et al., 1992). Patrones reproductivos evaluados en campo y en laboratorio, demuestran que el ciclo reproductivo para $A$. jamaicensis debe estar bajo control genético, 
sobretodo durante el segundo estro postparto (Wilson et al., 1991)

Aun no está claro qué factores desencadenan el inicio del período de reproducción. Por ejemplo, para A. jamaicensis se ha señalado que debe estar influenciado por alguna señal medioambiental que reanude y mantenga la periodicidad del ciclo reproductivo cada año. Dos posibles "señales" han sido sugeridas, una de ellas es la precipitación y la otra un repentino cambio en la cantidad de recurso energético disponible, pero debido a que el segundo período de nacimientos para $A$. jamaicensis ocurre en plena estación lluviosa ambas han sido descartadas (Wilson et al., 1991). En el Perú la mayoría de murciélagos, especialmente frugívoros, tienen crías durante la estación húmeda (Graham, 1989), por tal razón el parámetro ambiental más crítico para la reproducción de murciélagos ha recaído en la estacionalidad del patrón de lluvias, la cual afecta los ciclos reproductivos a través del suplemento alimenticio (Wilson, 1979); sin embargo, éste no es el caso para el área de estudio. En general, la costa del Pacífico es caracterizada por una baja precipitación anual $(<50 \mathrm{~mm})$, la mayoría de la cual cae entre diciembre y abril, período en el cual se reproducen las especies evaluadas en este estudio. Graham (1989) señala que en la costa durante la "estación húmeda" (octubre - abril), un $60 \%$ de especies de murciélagos se encuentran en reproducción; tendencia que ha sido observada en G. soricina y A. fraterculus durante este estudio. Observaciones de condiciones reproductivas más detalladas y que incluyan variables tales como temperatura y humedad relativa, deben confirmar el efecto de estas sobre la reproducción; el área de estudio puede ofrecer un interesante oportunidad de evaluar dicho efecto, ya que la variable precipitación esta aislada.

Graham (1989) encontró que en el Perú, los registros de nacimientos y destetes durante la estación seca es mayor en las especies nectarívoras que en las frugívoras; así mismo señaló que los nacimientos durante la estación seca deben ser la regla para los murciélagos que consumen néctar, pero la excepción para los frugívoros; dicha regla se invierta durante la estación húmeda. Estos son patrones predecibles, dado que ambos grupos presumiblemente experimentan variación estacional en sus suplementos alimenticios; pues el recurso floral es mayor durante la estación seca, por lo menos en el bosque lluvioso (Terborgh, 1983). Un desfase similar al mencionado en los períodos reproductivos de nectarívoros y frugívoros, ha sido observado en nuestra evaluación (Figura 4), es decir primero se reproduce $G$. soricina y luego $A$. fraterculus; sin embargo, éste patrón debe ser confirmado con un estudio adicional de fenología de plantas susceptibles de ser visitadas por murciélagos. Probablemente la conducta reproductiva siga un patrón coincidente con las estaciones o cambios en la temperatura o humedad relativa, los cuales podrían determinar una mejor oferta de alimento con picos de abundancia entre enero - mayo, meses de reproducción para ambas especies.

El patrón de desfase mencionado en el párrafo anterior, también podría sugerir una dieta de $G$. soricina principalmente constituida por néctar, ya que sus picos de reproducción anteceden al de un frugívoro (A. fraterculus), es decir en la época de mayor abundancia de flores. Sin embargo, también es necesario conocer los hábitos alimentarios de esta especie para comprobar dicha hipótesis; considerando también algunas diferencias alimentarias a nivel sexual, la cual es más marcada durante la época de reproducción (Alvarez \& Sánchez-Casas, 1999). Así mismo, también hay que considerar la dieta de $G$. soricina la cual es muy variable, dependiendo del área y la latitud (Nowak, 1999).

De las otras especies no se tienen registros de reproducción, pero se puede esperar algún tipo de comportamiento. Por ejemplo, Anoura geoffroyi que es considerado principalmente insectívoro y consumidor de néctar y polen (Nowak, 1999) presenta un comportamiento reproductivo monoestro estacional (Eisenberg \& Redford, 1999) y debe adecuar su período de reproducción al de su pariente glosofagíneo G. soricina, para evitar algún tipo de competencia por alimento. De Lasiurus blossevillii se conoce que es el único género de murciélagos que tienen más de dos crías por año y posee hábitos insectívoros (Nowak, 1999).

Los patrones reproductivos encontrados en $A$. fraterculus y G. soricina son ampliamente sugestivos. Sin embargo, las observaciones de individuos marcados de edad conocida serán necesarios para determinar la edad precisa en la cual la madurez sexual es alcanzada en ambas especies. Así mismo, sería pertinente identificar si existe algún patrón de retraso en el desarrollo embrionario, tal como ocurre en A. jamaicensis.

\section{Agradecimientos}

A todos los que colaboraron en el estudio de campo, especialmente a Raúl Acosta, Paola Aibar, Jessica Amanzo y Dante Pérez colegas de la UNALM. Así mismo a la bióloga Zulema Quinteros por su ayuda con los permisos para realizar las evaluaciones en el campus de la UNALM. A Sergio Solari y Lucía Luna del Museo de Historia Natural de la Universidad Nacional Mayor de San Marcos por sus comentarios y aportes a esta investigación y; a las autoridades de la Universidad Nacional Agraria La Molina por los permisos otorgados para las evaluaciones de campo.

\section{Literatura citada}

Alvarez T. \& Sánchez-Casas N. 1999. Diferenciación alimentaria entre los sexos de Glossophaga soricina (Chiroptera: Phyllostomidae) en México. 
Tropical Biology On Line, Revista de Biologia Tropical. 47 Issue 4.

Ascorra C., Gorchov D. \& Cornejo F. 1989. Observaciones en aves y murciélagos relacionadas con la dispersión de semillas en el valle del Palcazú, selva central del Perú. Boletín de Lima. $\mathrm{N}^{\circ} 62: 91-96$.

Ascorra C., Gorchov D. \& Cornejo F. 1993. The bats from Jenaro Herrera, Loreto, Peru. Mammalia. t. 57(4).

Ascorra C., Solari S. \& Wilson D. 1996. Diversidad y ecología de los quiropteros en Pakitza. Pages 593612 in Manu: The Biodiversity of Southeastern Perú. (D. E. Wilson and A. Sandoval, eds.) Smithsonian Institution Press and Ed. Horizonte, Lima.

August P.V. \& Baker R.J. 1982. Observations on the reproductive ecology of some Neotropical bats. Mammalia. 46: 177-181.

Autino A.G. \& Barquez R.M.. 1993. Patrones reproductivos y alimenticios de dos especies simpatricas del genero Sturnira (Chiroptera, Phyllostomidae). Mastozoología Neotropical. 1 (1): 73-80.

Bonaccorso F.J. 1979. Foraging and reproductive ecology in a Panamanian bat community. Bull. Fla. State. Mus. Biol. Ser. 24: 359-408.

Bradbury J.W. \& Vehrencamp S.L.. 1976. Social organization and foraging in emballonurid bats. I. Field studies. Behavioral Ecology and Sociobiology. 1: 337-381.

Dinerstein E. 1986. Reproductive Ecology of Fruit Bats and the Seasonality of Fruit Production in a Costa Rican Cloud Forest. Biotropica. 18(4): 307318.

Eisenberg J.F. \& Redford K.H. 1999. Mammals of the Neotropics. The Central Neotropics, volume 3 Ecuador, Peru, Bolivia, Brazil. The University of Chicago Press.

Emmons L.H. \& Feer F. 1997. Neotopical Rainforest Mammals, a field guide. Second Edition. The University of Chicago Press. Chicago.

Fenton, M.B., Acharya L., Audet D.,Hickey M.B.C., Merriman C., Obrist M.K., Syme D.M., \& Adkins B. 1992. Phyllostomid btas (Chiroptera: Phyllostomidae) as indicators of habitat disruption in the Neotropics. Biotropica. 24: 440-446.

Fleming T.H., Hooper E.T., \& Wilson D.E. 1972. Three Central American bat comumnities:estructure, reproductive cycles and movement patterns. Ecology. 53: 653-670.

Frankie G.H., Baker H. \& Opler P.A. 1974. Comparative phenological studies of trees in tropical wet and dry forests in the lowlands of Costa Rica. J. Ecol. 62: 881-919.

Gardner A. 1977. feeding habits. In R. J. Baker, J. K. Jones \& D.C. Carter (Eds). Biology of bats in the New World family Phyllostomidae, Part II, pp
293-350. Spec. Publ. Mus Texas Tech Univ. Lubbock.

Graham G.L. 1989. Seasonality of Reproduction in Peruvian Bats. In "Studies in Neotropical Mammalogy: Essays in Honor of Philip Hershkovitz" (Bruce D. Patterson and Robert M. Timm, eds). Fieldiana Zoology. 39: 173-186.

Hilty S.L. 1980. Flowering and fruiting periodicity in a premontane rain forest in pacific Colombia. Biotropica. 12: 292 - 306.

Holdridge L. 2000. Ecologia basada en zonas de vida. Quinta reimpresión. Instituto Interamericano de cooperación para la agricultura. San José, Costa Rica.

Howe H.F. \& Smallwood J. 1982. Ecology of seed dispersal. Ann. Rev. Ecol. Syst. N ${ }^{\circ} 13$, pp 201228.

Humphrey S.R. \& Bonaccorso F.J.. 1979. Population and community ecology. In: Baker, R.J., J.K. Jones, Jr. \& D.C. Carter (Eds.), Biology of Bats of the New World Family Phyllostomidae. Part II, Special Publications, Museum, Texas Tech University. 16: 409-441.

Koopman K.F. 1978. Zoogeography of Peruvian Bats with Special Emphasis on the Role of the Andes. American Museum Novitates 2651: 1-33.

Laval R.K. \& H.S. Fitch. 1977. Notes on some Costa Rican bats. Brenesia Dep. Hist Nat. Mus. Nac. Costa Rica, 10/11: 77-83.

Nowak R.M. 1999. Walker's mammals of the world. Sixth Ed. Vol. 1. The Johns Hopkins Univ. Press. Baltimore and London.

Ortiz de la Puente, J. 1951. Estudio monográfico de los quirópteros de Lima y alrededores. Serie A. Zoología. Publ. Mus. His. Nat. UNMSM (A) 7:148.

Pacheco V., Paredes B., Crosby J., Gayoso J., Cardoso F. y Sisniegas H. 1990. Notas sobre la avifauna en ambientes urbanos y sugerencia sobre planeamiento de parques. Serie A. Zoología. Publ. Mus. His. nat. UNMSM (A). 35:1-7.

Patterson B.D., Pacheco V. \& Ashley M.V. 1992. On the origins of the western slope region of endemism: Systematics of fig-eating bats, Genus Artibeus. Memorias del Museo de Historia Naturalm UNMSM (Lima). 21:189-205.

Racey P.A. 1982. Ecology of bat reproduction, pp 57104. In Kunz, T. H., ed., Ecology of Bats. Plenum Press, New York.

Romano M.C., Maidagan J.I. \& Pire E.F. 1999. Behavior and demography in an urban colony of Tadarida brasiliensis (Chiroptera: Molossidae) in Rosario, Argentina. Tropical Biology On Line, Revista de Biologia Tropical, 47 (4).

Solari S., Vivar E., Rodríguez J.J. \& Mena J.L.. 1998. Small Mammals: Biodiversity Assessment in the Lower Urubamba Region, Cusco, Peru. Pages 209218 in Biodiversity Assessment and Long Term 
Monitoring of the Lower Urubamba Region, Cusco, Peru: Cashiriari-Well Site and the Camisea and Urubamba Rivers. SI/Mab Series \#2 (F. Dallmeier and A. Alonso, eds.). Smithsonian Institution/ MAB Biodiversity program, Washington, D.C., USA.

Terborgh J. 1983. Five New World Primates. Princeton University Press, Princeton, N. J.

Voss R.S. \& Emmons L.H. 1996. Mammalian Diversity in Neotropical Lowland Rainforests: A preliminary Assessment. Bulletin of the American Museum of Natural History, $\mathrm{N}^{\circ} 230$, New York.

Wilson D.E. 1979. Reproductive patterns. Pp. 317-378 in Biology of bats of the New World Family Phyllostamatidae. Part III (R. J. Baker, J. K. Jones,
Jr., y D. C. Carter, eds.). Special Publications, The Museum Texas University. 16: 1-441.

Wilson D.E., Handley C.O. Jr. \& Gardner A.L. 1991. Reproduction on Barro Colorado Island. In Demography and natural history of the common fruit bat, Artibeus jamaicensis, on Barro Colorado Island, Panama (Handley, C. O. Jr.; D. E. Wilson, \& A. L. Gardner eds.) Smithsonian Contrib. Zool. 511:1-173.

Wilson D.E., Ascorra C.F. \& Solari S. 1996. Bats as Indicators of Habitat Disturbance. Pages 613-625 in Manu: The Biodiversity of Southeastern Perú. (D. E. Wilson and A. Sandoval, eds.) Smithsonian Institution Press and Ed. Horizonte, Lima. 